\title{
Stored Energy and the Charging Energy Efficiency in a Memcapacitor Circuit
}

\author{
Fatih Tulumbaci $^{1}$, Suayb Cagri Yener $^{2,3}$, Resat Mutlu ${ }^{4}$ \\ ${ }^{1}$ Electronics Department, Corlu Vocational School, Tekirdag Namik Kemal University \\ 59860, Corlu,Tekirdag, Turkey \\ ftulumbaci@nku.edu.tr; \\ ${ }^{2}$ Department of Electrical Electronics Engineering, Sakarya University \\ 54050, Serdivan, Sakarya, Turkey \\ ${ }^{3}$ Electromagnetics Research Center, Sakarya University \\ 54050, Serdivan, Sakarya, Turkey \\ syener@sakarya.edu.tr \\ ${ }^{4}$ Electronics and Communication Engineering Department, Corlu Engineering Faculty, Tekirdag Namik Kemal University \\ 59860, Corlu, Tekirdag, Turkey \\ rmutlu@nku.edu.tr
}

\begin{abstract}
Memcapacitor is a nonlinear circuit component. It has similar properties to a capacitor but it also shows different properties. To the best of our knowledge, a memcapacitor's stored energy has not been investigated in literature yet. In this work, it has been shown that while a capacitor's energy can be calculated using just its voltage and capacitance, it cannot for a memcapacitor. In this work, a memcapacitor modeled with a simple memcapacitance with a linear flux dependency and a saturation mechanism is charged with a dc voltage source with a series resistance. Its energy is calculated using simulations It has been shown that the equations describing charging of the memcapacitor are quite nonlinear and numerical methods should be employed for calculation of its charge and stored energy. Also it has been shown that its charging efficiency might be higher or lower than a LTI capacitor.
\end{abstract}

Keywords: Memcapacitor, Memcapacitive Systems, Stored Energy, Energy Efficiency.

\section{Introduction}

In 1971, L.O. Chua predicted that another fundamental circuit element called memristor should have existed [1]. In 1976, Chua and Kang claimed that systems called memristive systems showing similar properties to memristors exist and they showed a memristive system and a memristor under AC exciation should have a zero-crossing current-voltage hysteresis curve in 1977 [2]. Decades later, an HP research team announced that they found the missing fundamental circuit element, memristor [3]. Memristor and memristive systems have become a hot research topic nowadays [4-5]. In 2009, Ventra et al claimed that there should have been circuit elements capacitors and inductors with memories and called them memcapacitor and meminductor and they also described memcapacitive and meminductive systems in [6]. Research on ionic and solid-state memcapacitors are exist in literature [7-8]. Some materials and devices are reported to have memcapacitive properties [7-16]. A bistable non-volatile elastic membrane memcapacitor is shown to exhibite chaotic behavior in [17]. It is shown that memcapacitor can be used in chaotic oscillators [18-20]. An inductor-memcapacitor oscillation circuit is examined in [21]. Some memcapacitor studies have focused on designing memcapacitor emulator circuits $[22,23]$. Some of the memcapacitor research has focused on its properties, analysis, modeling and teaching [21,2327]. A review on memcapacitors can be found in [9]. Charging and discharging of a memcapacitor is inspected in [28]. A circuit with a capacitor and memcapacitor have been examined in [29]. Although, memcapacitor is not commercially available right now. The direction of research shows that at one point in the future they may come out in the market. There is a possibility that it may also emerge as power capacitors or back-up capacitors or memory elements. We should be ready to make the best use of it and this can only be done by knowing the element better and indicating its possible usage areas. While memristor is a dissipative element [1], meminductor and memcapacitor are not since they are similar to inductor and capacitor. Stored energy formula in a linear time-invariant (LTI) capacitor is well-known and tought in high school. However, to the best of our knowledge, it has not been investigated yet how to calculate the stored energy in a 
memcapacitor. In this paper, a circuit consisting of a dc voltage source, a series resistance, and a memcapacitor is studied and a methodology has been given to calculate the energy of the memcapacitor. In this paper, a flux dependent memcapacitor model, which is similar to those used in $[21,24,29]$, has been used for simulations. However, a saturation mechanism is added to the memcapacitor model used in this paper. The charging efficiency of a LTI capacitor from a dc voltage source is $50 \%$ regardless of its series resistor [30]. The memcapacitor charge efficiency have also been examined considering both positive and negative polarities of the memcapacitor used in this study.

The remainder of the paper is organized as follows. In the second section, the memcapacitor model used in this paper is given. In the third section, the charging of a capacitor by a DC Supply with a series resistor and its stored energy is reviewed. In the fourth section, it is shown that it is not possible to find a stored energy formula for a memcapacitor as simple as an LTI capacitor's. In the fifth section, charging of the memcapacitor with a constant voltage source considering for both of the polarities is simulated and also the simulation results for current, flux, charge, and voltage are given. In the sixth section, the memcapacitor's stored energy and its charging efficiency are calculated numerically for both of its polarities by the postprocessing of the simulation data. The paper is finished with the conclusion section.

\section{Flux-dependent Memcapacitor Model}

In [6], Ventra et al have described an $\mathrm{n}^{\text {th }}$ order voltage-controlled (voltage-dependent) memcapacitive system as

$$
\begin{gathered}
q(t)=C_{m}\left(x, V_{C}, t\right) V_{C}(t) \\
\frac{d x}{d t}=f\left(x, V_{C}, t\right)
\end{gathered}
$$

Where $q(t)$ is the charge on the memcapacitive element at time $t, V_{C}(t)$ is the memcapacitive element voltage, $x$ is a vector representing $\mathrm{n}$ internal state variables, and $C_{m}$ is the memcapacitance (short for memory capacitance), which depends on the state of the system.

In addition, they have defined a subclass of the memcapacitive systems with memcapacitance being only a function of the memcapacitor flux, and called them voltage-controlled memcapacitors in [6]. For a memcapacitor, Eqs. (1) and (2) reduce to

$$
q(t)=C_{m}(\lambda) V_{C}(t)
$$

and

$$
\frac{d \lambda(t)}{d t}=V_{C}(t)
$$

Where $C_{m}(\lambda)$ is the flux-dependent memcapacitor memcapacitance, $q(t)$ is the memcapacitor charge, $V_{C}(t)$ is the memcapacitor voltage, and $\lambda(t)$ is the memcapacitor flux, which is the integral of memcapacitor voltage by respect to time. Memcapacitor flux can be calculated as

$$
\lambda(t)=\int_{-\infty}^{t} V_{C}(\tau) d \tau \quad \text { or } \quad \lambda(t)=\int_{0}^{t} V_{C}(\tau) d \tau+\lambda_{0}
$$

Where $\lambda_{0}$ is the initial memcapacitor flux at time being zero. Taylor series are used to present memcapacitance in $[21,24]$. Using Mclaurin series, the memcapacitor's memcapacitance can be expressed as

$$
\mathrm{C}_{\mathrm{m}}(\lambda)=C(0)+\sum_{k=1}^{\infty} \frac{\partial C^{\mathrm{k}}(0)}{\partial q^{k}} \frac{\lambda^{\mathrm{k}}}{\mathrm{k} !}
$$

Ignoring the second or more order terms of Eq. (6) as done in $[21,24]$, the memcapacitance can be taken as

$$
\mathrm{C}_{\mathrm{m}}(\lambda) \cong C_{0}+\frac{\partial C(0)}{\partial q} \lambda
$$

Designating 


$$
C_{0}=C(0) \text { and } K=\frac{\partial C(0)}{\partial q}
$$

the memcapacitor's memcapacitance can be written as

$$
C_{m}(\lambda)=C_{0}+K \lambda(t)
$$

Now, let's take a look at the circuit in Figure 1.a. The memcapacitor polarity in this figure is called positive polarity and, therefore, the memcapacitor has positive polarity therein. The memcapacitor polarity in Figure 1.b is called negative polarity and, therefore, the memcapacitor has negative polarity therein.

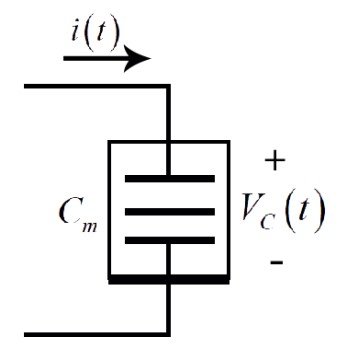

a)

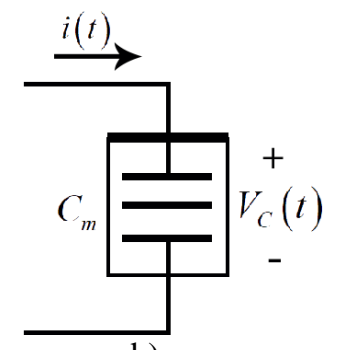

b)

Fig. 1: a) Memcapacitor with a Positive Flux Coefficient or with Positive Polarity. b) Memcapacitor with a Negative Flux Coefficient or with Negative Polarity.

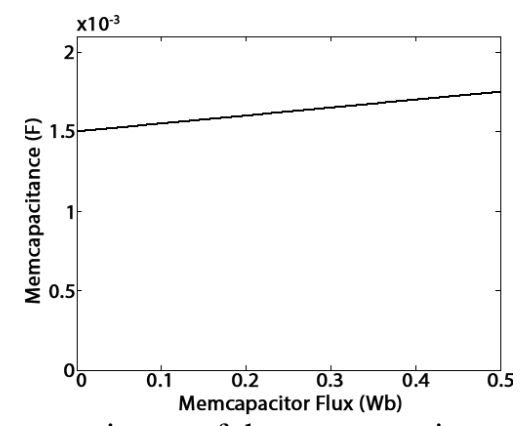

Fig. 2: Memcapacitance of the memcapacitor as function of memcapacitor-flux if the memcapacitor is not saturated.

There is a saturation mechanism in [6]. It is possible that every memcapacitor has some kind of saturation mechanism. That's why a saturation mechanism is incorporated into the model. The memcapacitor model is modified considering the polarities shown in Figure 1 as the following. At $\lambda=\lambda_{S A T}$, the memcapacitor memcapacitance becomes maximum and it is equal to

$$
C_{\max }=C_{0}+K \lambda_{S A T}
$$

If the memcapacitor has the positive polarity shown in Figure 1.a. and the memcapacitance becomes maximum, ie. $C=C_{\max }$, the memcapacitor stays constant and equal to $C_{\max }$ as long as the voltage applied stays positive. If the memcapacitor has the reverse polarity shown in Figure 1.b. and the memcapacitor memcapacitance becomes minimum, i.e. $C=C_{0}$ or and $\lambda=0$, the memcapacitor stays constant and equal to $C_{0}$ as long as the voltage applied stays negative. If the memcapacitor is not saturated, ie. $0<\lambda<\lambda_{S A T}$, the unsaturated memcapacitance function which is used in this paper is given in Figure 2.

\section{A LTI Capacitor Charged by a DC Voltage Source with a Resistor and its Stored Energy}

An LTI capacitor supplied by a DC voltage source is shown in Figure 3. The solution of the circuit in Figure 3 is wellknown. The circuit current, the capacitor voltage and the capacitor energy are respectively equal to

$$
\begin{gathered}
i_{C}(t)=\frac{V_{D C} e^{-t / \tau}}{R} \\
V_{C}(t)=V_{D C}\left(1-e^{-t / \tau}\right) \\
E_{C}=\frac{C_{p} V_{C}^{2}}{2}
\end{gathered}
$$

Where $V_{D C}$ is the voltage of the DC voltage source, $R$ is the resistance value in the circuit and $C_{p}$ is the capacitance of the LTI capacitor. 
In [30], it is shown that the charging capacitor voltage from zero to the supply voltage, the required energy of the capacitor is equal to the sum of the energy loss dissipated within the resistor and the energy radiated into space. In [30], it is also shown that the energy needed is not dependent on the series resistor shown in Figure 3. Series resistor might even be time-dependent however the result would not change. Its charging energy efficiency is always equal to $50 \%$, ie. The same amount of energy is wasted to charge the capacitor from a constant voltage source.

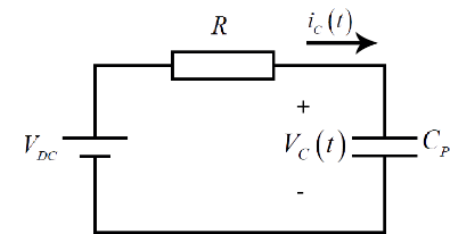

Fig. 3: Capacitor Supplied a Constant Voltage Source.

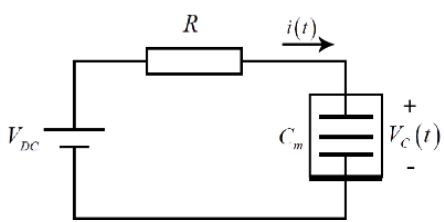

a)

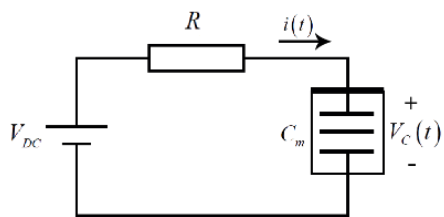

b)

Fig. 4: Charging of a memcapacitor by a DC voltage source with a series resistor with the memcapacitor having a) positive and b) negative polarity.

\section{On The Calculation of a Memcapacitor's Energy}

In this section, first, we try to calculate the stored energy of a memcapacitor. The memcapacitor current is calculated by taking the derivative of the memcapacitor charge:

$$
i_{C}(t)=\frac{d q(t)}{d t}=\frac{d\left(C_{m} V_{C}\right)}{d t}=\frac{d C_{m}}{d t} V_{C}+C_{m} \frac{d V_{C}}{d t}=\frac{d C_{m}}{d \lambda} \frac{d \lambda}{d t} V_{C}+C_{m} \frac{d V_{C}}{d t}
$$

Using the relationship between the memcapacitor voltage and flux,

$$
\mathrm{V}_{\mathrm{C}}=\frac{\mathrm{d} \lambda}{\mathrm{dt}}
$$

Eq. (14) turns into

$$
i_{C}(t)=\frac{\partial \mathrm{C}_{\mathrm{m}}}{\partial \lambda} \mathrm{V}_{\mathrm{C}}^{2}+\mathrm{C}_{\mathrm{m}} \frac{\mathrm{dV}_{\mathrm{C}}}{\mathrm{dt}}
$$

The memcapacitor power is

$$
P_{C}=V_{C} i_{C}=V_{C} \frac{d\left(C_{m} V_{C}\right)}{d t}
$$

Then, the memcapacitor energy can be expressed as

$$
E_{C}=\int_{t=0}^{t} P_{C} d t=\int_{t=0}^{t} V_{C}\left(\frac{\partial C_{m}}{\partial \lambda} V_{C}^{2}+C_{m} \frac{d V_{C}}{d t}\right) d t=\int_{t=0}^{t}\left(\frac{\partial C_{m}}{\partial \lambda} V_{C}^{3}+C_{m} V_{C} \frac{d V_{C}}{d t}\right) d t
$$

Remembering that memcapacitance is a function of memcapacitor flux, which is the integration of memcapacitor voltage, the integral given in Eq. (18) is not solvable since the flux is dependent on the integration of voltage by respect to time. Therefore, the integral in Eq. (18) is path-dependent and a memcapacitor's energy cannot be found as just a function of memcapacitor voltage and memcapacitor parameters as in the case of a linear time-invariant capacitor. That's why numerical integration methods should be employed for its energy calculation. The circuit is solved numerically in the next section and the memcapacitor energy is calculated in the sixth section.

\section{Charging Memcapacitor with a Constant Voltage Source Considering both Polarities}

Now, let's take a look at the circuits in Figure 4.a and 4.b. The memcapacitor polarity is positive in Figure 4.a and negative Figure 4.b. Using Kirchoff's Voltage Law;

$$
V_{D C}=V_{R}+V_{C}=i R+\frac{q}{C_{m}}
$$


Where $V_{R}$ and $i$ are the voltage and current of the resistor. Using Kirchoff's Current Law; the resistor current is equal to memcapacitor current;

$$
i=i_{C}
$$

Using Equation (14),

$$
V_{D C}=R \frac{\partial C_{m}}{\partial \lambda} V_{C}^{2}+R C_{m} \frac{d V_{C}}{d t}+V_{C}
$$

Remembering the Eq. (8), equation (21) turns into

$$
V_{D C}=R K V_{C}^{2}+R\left(C_{0}+K \lambda\right) \frac{d V_{C}}{d t}+V_{C}
$$

Using the relationship between the memcapacitor voltage and flux, ie. Eq. (4),

$$
V_{D C}=R K\left(\frac{d \lambda}{d t}\right)^{2}+R\left(C_{0}+K \lambda\right) \frac{d^{2} \lambda}{d t^{2}}+\frac{d \lambda}{d t}
$$

An analytical solution for Eq. (23) is not possible. That's why a numerical method must be used to simulate the charging of the memcapacitor. Simulink toolbox of Matlab is used to solve the differential equation numerically using Range-Kutta method. The system is simulated with the memcapacitor having positive polarity for the parameters given in Table 1. The memcapacitor's initial charge is taken zero. The memcapacitor's flux is taken to be zero for positive polarity and $\lambda_{S A T}$ for negative polarity. Memcapacitance, charge, current, flux, and voltage of the memcapacitor is shown in Figures 5-9 when the memcapacitor for the positive and negative polarities. The voltage of the memcapacitor increases during transient and it reaches to the dc supply voltage at steady-state. If the memcapacitor is not saturated, its flux and, therefore, memcapacitance varies by time, too. The saturation can also be seen in Figures 5-9 for both of the polarities.

Table 1: The circuit Parameters and Memcapacitor Initial Conditions.

\begin{tabular}{|c|c|c|c|c|c|c|}
\hline Parameter & $K$ & $C_{0}$ & $C_{S A T}$ & $q(0)$ & $V_{D C}$ & $R$ \\
\hline Value & $0.5 \mathrm{mF} / \mathrm{Wb}$ & $1.5 \mathrm{mF}$ & $2.5 \mathrm{mF}$ & $0.05 \mathrm{C}$ & $10 \mathrm{~V}$ & $10 \Omega$ \\
\hline
\end{tabular}

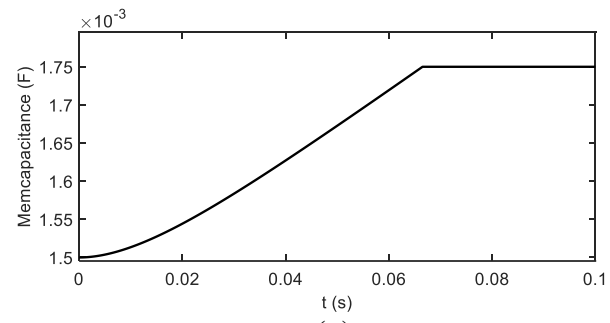

(a)

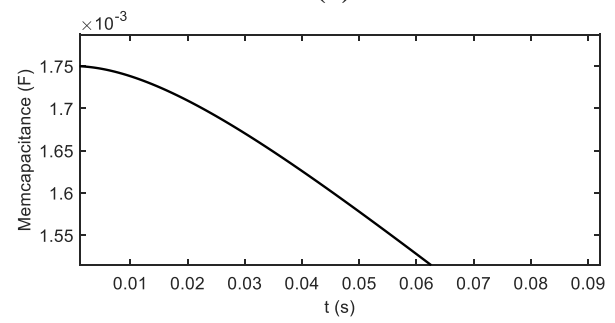

(b)

Fig. 5: The Memcapacitor

Memcapacitance vs Time for a) positive and b) negative polarity.

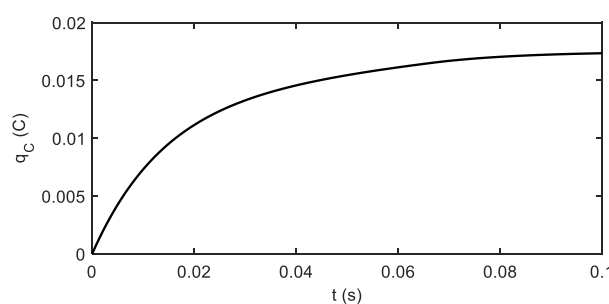

(a)

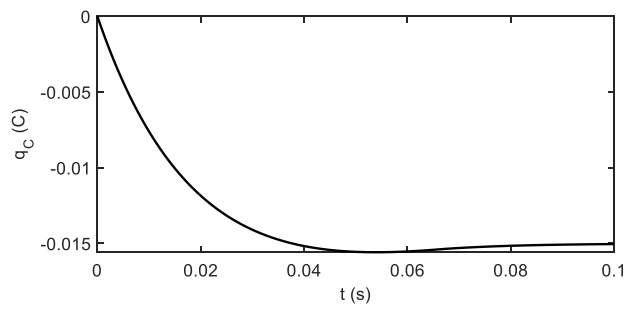

(b)

Fig. 6: The Memcapacitor Charge vs Time for a) positive and b) negative polarity.

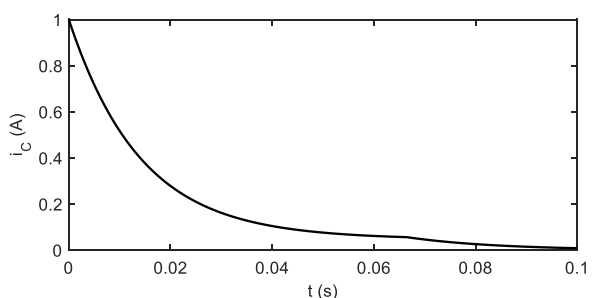

(a)

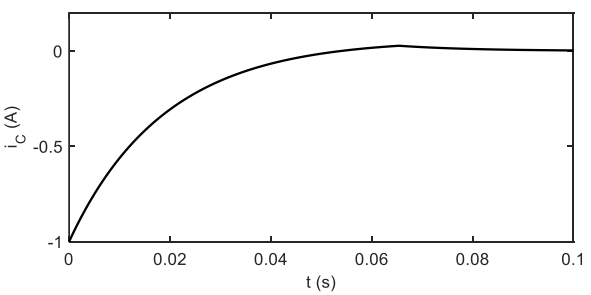

(b)

Fig. 7: The Memcapacitor Current vs. Time for a) positive and $b$ ) negative polarity. 


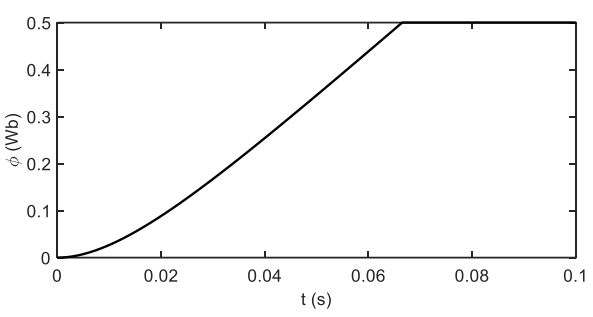

(a)

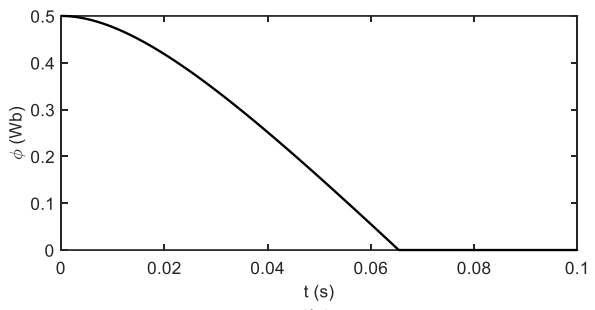

(b)

Fig. 8: The Memcapacitor Flux vs. Time for a) positive and b) negative polarity.

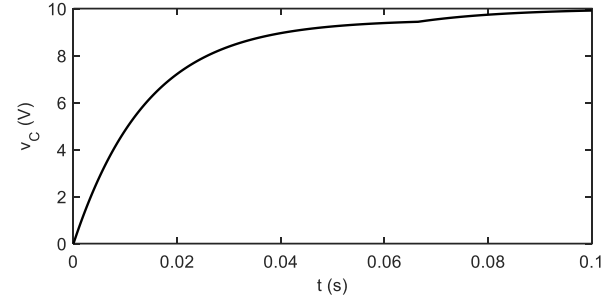

(a)

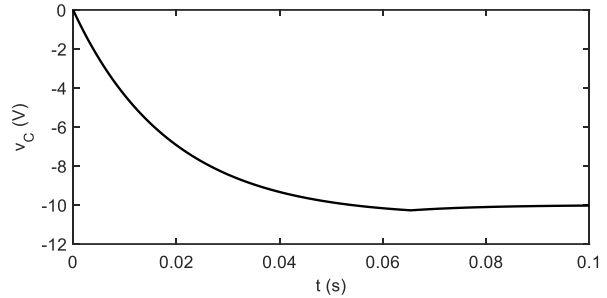

(b)

Fig. 9: The Memcapacitor Voltage vs. Time for a) positive and b) negative polarity.

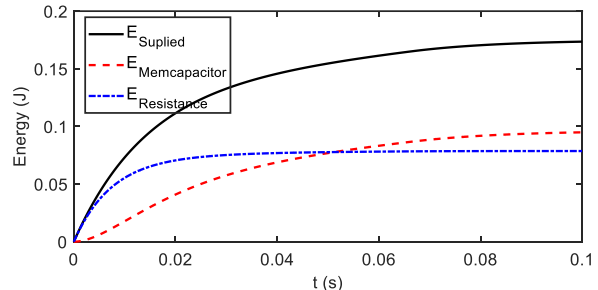

(a)

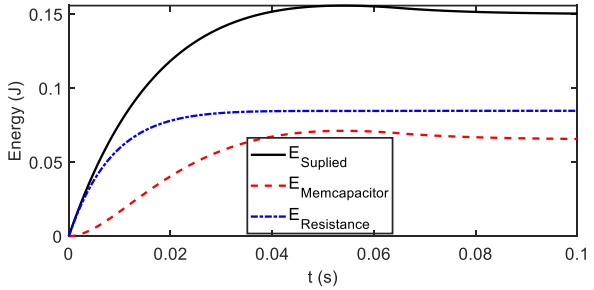

(b)

Fig. 10: The Supplied Energy, the Energy Loss and the Stored Energy for a) positive and $b$ ) negative polarity.

\section{Calculation of the Stored Energy of a Memcapacitor and its Comparison to that of an LTI Capacitor Considering Both Polarities}

In this section, the stored energy in the memcapacitor, the energy loss within the equivalent series resistor, and the energy supplied by the source are calculated postprocessing the simulation data obtained before. The stored energy is

$$
E_{\text {STORED }}=\int_{t=0}^{t=T_{\text {last }}} V_{C} d q_{C}
$$

where $T_{\text {last }}$ is the simulation time. The energy loss within the equivalent series resistor is

$$
E_{\text {LOSS }}=\int_{t=0}^{t=T_{\text {last }}} i^{2} R d t
$$

The energy supplied by the source is

$$
E_{S}=\int_{t=0}^{t=T_{\text {last }}} V_{D C} d q_{C}=V_{D C} \int_{t=0}^{t=T_{\text {last }}} d q_{C}=V_{D C}\left(q\left(T_{\text {last }}\right)-q(0)\right)
$$

The energy Efficiency of the charging process is

$$
\eta=\frac{E_{\text {STORED }}}{E_{S}}=\frac{E_{C}}{E_{S}}
$$

For positive and negative polarity, the energy loss within the resistor, the energy stored by the memcapacitor, and the energy supplied by the source are simulated and shown in Figure 10. The charging efficiency of the memcapacitor for positive and negative polarity are shown in Figure 11 and it has been found that the charging efficiency is dependent on the memcapacitor polarity and might be higher or lower than $50 \%$ while it is exactly $50 \%$ for an LTI capacitor. The charging efficiency is found to be $54.65 \%$ for positive polarity and $42.19 \%$ for negative polarity. This is an impotant finding if memcapacitors are to be used as a memory element to store data. The energy loss in a memcapacitor or a memcapcitive system is more complex. 


\section{Conclusion}

Memcapacitor is an elusive circuit element yet and its usage with other circuit elements should be examined in detail so that it can be exploited more efficiently. In this work, charging of a memcapacitor with a simple saturable memcapacitance and a linear flux dependency from a DC voltage source is simulated and the polarity dependent energy losses of the circuit and the memcapacitor's stored energy are examined from an energy efficiency point of view and calculated numerically. It has been shown that the equation describing the memcapacitor's charging is quite nonlinear, it has no exact solution and numerical methods should be employed for calculation of its circuit waveforms and stored energy. The saturation mechanism of the memcapacitor also makes transients more complex. It has been found out that the time-varying memcapacitance makes finding an energy formula as simple as the case for an LTI capacitor for the memcapacitor not possible, it is not sufficient to know a memcapacitor's voltage for that purpose, and, therefore, numerical methods should be employed for its calculation.

The energy efficiency of a circuit element is an important concept and becoming more and more important while energy sources are being depleted on the world. We have shown that the energy efficiency of charging memcapacitors might be different than linear time-invariant capacitors. In some cases, it may be advantageous to use memcapacitors if such a device emerges in the market in the future. The charging efficiency of the memcapacitor circuit is found to be polarity dependent while the energy loss of the circuit consisting of a LTI capacitor, a resistor and a DC voltage source does not. However, for charging an LTI capacitor, it is not so. Also, the charging energy efficiency of an LTI capacitor supplied by a dc voltage source is always $50 \%$ while that of a memcapacitor might be more or less than that.

In this study, the estimation of the energy stored in a memcapacitor is recognized as a problem since it is not sufficient to read its voltage for the prediction of its energy due to the flux-dependence of the memcapacitance of a memcapacitor. Estimators (electronics circuits) may be needed for that purpose. It is a challenge for observer or estimator designers and circuit theoreticians. Here, we suggest two methods for that purpose. The first method estimates the energy of the memcapacitor systems numerically by reading its current and voltage as done in the simulations in this study. Also, a second method is a hardware solution and an estimator circuit uses a reading pulse by means of using an inductor can also be used. Such a method would also consume energy but the energy it consumes might possibly be ignored especially for high energy applications. Due to the space considerations, the methods are told further here and left as future work.

The memcapacitors are a subset of the memcapacitive systems whose energy estimation might be more complex than memcapacitors. However, the experience gained here can be used to calculate their energy by most-probably employing numerical methods, too.

Memcapacitor is a quite mysterious element. When more memcapacitor data become available in literature, the energy of the memcapacitors should also be investigated more for a better understanding of the circuit element memcapacitor so that it can be used more effectively for its possible applications such as memories and energy storage.

\section{References}

[1] L. O. Chua, "Memristor - The Missing Circuit Element," IEEE Trans. Circuit Theory, vol. 18, pp. 507-519, 1971.

[2] L. O. Chua and S. M. Kang, "Memristive devices and systems," Proc.IEEE, vol. 64, pp. 209-223, 1976.

[3] D. B. Strukov, G. S. Snider, D. R. Stewart, and R. S. Williams, "The missing memristor found," Nature (London), vol. 453, pp. 80-83, 2008.

[4] The fourth element: characteristics, modeling and electromagnetic theory of the memristor, O. Kavehei, A. Iqbal, Y.S. Kim, K. Esraghian, S.F. AL-Sarawi and D. Abbott, Proc. R. Soc. A, publishes.

[5] Mazumder, P., Kang, S. M., \& Waser, R., "Memristors: devices, models, and applications." Proceedings of the IEEE, 100(6), 1911-1919, 2012.

[6] M. Di Ventra ,Yu. V. Pershin and L. O. Chua "Circuit elements with memory: memristors, memcapacitors and meminductors" Proc. IEEE, vol. 97, pp. 1717-1724, 2009.

[7] Martinez-Rincon, J., Di Ventra, M., \& Pershin, Y. V. (2010). Solid-state memcapacitive system with negative and diverging capacitance. Physical Review B, 81(19), 195430.

[8] Krems, M., Pershin, Y. V., \& Di Ventra, M., "Ionic Memcapacitive Effects in Nanopores" Nano ACS Publications, 
2010.

[9] Yuriy V. Pershin and Massimiliano Di Ventra, "Memory effects in complex materials and nanoscale systems", in Physics, v. 60, pages 145-227, 2011.

[10] Goldflam, M. D., Driscoll, T., Chapler, B., Khatib, O., Marie Jokerst, N., Palit, S., ... \& Ventra, M. D. (2011). Reconfigurable gradient index using VO2 memory metamaterials. Applied Physics Letters, 99(4), 044103.

[11] Singh, R. K., \& Mamta, K. An account of spin memristive and memcapacitive systems: Next generation memory devices. IOSR Journal of Applied Physics e-ISSN: 2278-4861.Volume 6, Issue 3 Ver. III (2014), PP 07-23

[12] Park, D., Yang, P., Kim, H. J., Beom, K., Lee, H. H., Kang, C. J., \& Yoon, T. S. (2018). Analog reversible nonvolatile memcapacitance in metal-oxide-semiconductor memcapacitor with ITO/HfOx/Si structure. Applied Physics Letters, 113(16), 162102.

[13] Khan, A. K., \& Lee, B. H. (2016). Monolayer MoS2 metal insulator transition based memcapacitor modeling with extension to a ternary device. AIP Advances, 6(9), 095022.

[14] Flak, J., \& Poikonen, J. K. (2014). Solid-state memcapacitors and their applications. In Memristor Networks (pp. 585-601). Springer, Cham.

[15] Shen, Y., Wang, G., Liang, Y., Yu, S., \& Iu, H. H. C. (2019). Parasitic memcapacitor effects on HP TiO2 memristor dynamics. IEEE Access, 7, 59825-59831.

[16] Sun, J., Lind, E., Maximov, I., Xu, H. Q., Memristive and Memcapacitive Characteristics of a Au/Ti-HfO2InP/InGaAs Diode. Electron Device Letters, IEEE, vol.32, no.2, pp.131-133, Feb. 2011.

[17] Bistable non-volatile elastic membrane memcapacitor exhibiting chaotic behavior J. Martinez-Rincon, Y. V. Pershin Comments: IEEE Transactions on Electron Devices.

[18] Hu, Z., Li, Y., Jia, L., \& Yu, J. Chaotic oscillator based on voltage-controlled memcapacitor. 2010 International Conference on Communications, Circuits and Systems (ICCCAS). IEEE, 2010. p. 824-827.

[19] Rajagopal, K., Akgul, A., Jafari, S., \& Aricioglu, B. (2018). A chaotic memcapacitor oscillator with two unstable equilibriums and its fractional form with engineering applications. Nonlinear Dynamics, 91(2), 957-974.

[20] Yuan, F., Wang, G., \& Wang, X. (2017). Chaotic oscillator containing memcapacitor and meminductor and its, dimensionality reduction analysis. Chaos: An Interdisciplinary Journal of Nonlinear Science, 27(3), 033103.

[21] Yener Ş. Ç., Mutlu R., Small signal model of memcapacitor-inductor oscillation circuit, Electric Electronics, Computer Science, Biomedical Engineerings’xx Meeting (EBBT), 2017 (20.04.2017-21.04.2017).

[22] Y. Babacan, "An Operational Transconductance Amplifier-based Memcapacitor and Meminductor." Electrica 18.1 (2018): pp. 36-38

[23] Radwan, A. G., \& Fouda, M. E. (2015). Memcapacitor: Modeling, Analysis, and Emulators. In On the Mathematical Modeling of Memristor, Memcapacitor, and Meminductor (pp. 151-185). Springer, Cham.

[24] Karakulak, E., Mutlu, R., Explanation of Hysteresis Curve of a Flux-dependent Memcapacitor (Memory-capacitor) Using Taylor Series and Parametric Functions. 6th International Advanced Technologies Symposium (IATS'11), pp.419-422, 16-18 May, 2011.

[25] Biolek, D., Biolek, Z., \& Biolkova, V. (2011). Pinched hysteretic loops of ideal memristors, memcapacitors and meminductors must be 'self-crossing'. Electronics letters, 47(25), 1385-1387.

[26] Fouda, M. E., Khatib, M. A., \& Radwan, A. G. (2013, December). On the mathematical modeling of series and memcapacitors. In 2013 25th International Conference on Microelectronics (ICM) (pp. 1-4). IEEE.

[27] Biolek, D., Biolek, Z., Biolková, V., Behavioral Modeling of Memcapacitor. Radioengineering, vol. 20, No. 1, pp. 228-233, April 2011.

[28] He, P., Wang, L., \& Duan, S. "The charging and discharging characteristics of memcapacitor storage with applications." Proceedings of the 10th World Congress on Intelligent Control and Automation. IEEE, 2012.

[29] Mutlu, R., Akin, O.Ç., The Memcapacitor-Capacitor Problem. 2nd International Symposium on Computing in Science \& Engineering (ISCSE), proc. no. 200/21, June 1-4, 2011.

[30] A.M. Abu-Labdeh, S.M. Al-Jaber, Energy consideration from non-equilibrium to equilibrium state in the process of charging a capacitor, J. Electrostat. (2008). 